\title{
Do the Physical Characteristics of a Virtual Reality Device Contraindicate Its Use for Balance Assessment?
}

\author{
Jason P. Mihalik, Luv Kohli, and Mary C. Whitton
}

\begin{abstract}
Context: Virtual reality environments may allow researchers to investigate functional balance performance without risks associated with testing in the real world. Objective: To investigate the effects of the mass of a head-mounted display (HMD) on balance performance. Design: Counterbalanced pretest-posttest. Setting: Virtual reality laboratory. Participants: 20 healthy college students. Intervention(s): Balance Error Scoring System (BESS) with a tracker-only headband and again with tracker plus HMD was performed. Main Outcome Measures: BESS error scores, elliptical sway area, and center of pressure travel distance were recorded. Results: No effect of the HMD mass on balance performance was observed. A significant stance by surface interaction was present but was negated when the HMD conditions were included in the model. Conclusions: The mass of a HMD has not been proven to adversely affect balance performance. These data suggest the HMD mass is not a contraindication to the use of immersive virtual environments in future concussion research involving balance. Keywords: mild traumatic brain injury, virtual environment, athletic injuries, postural stability, college
\end{abstract}

The management of sports-related concussion remains an area of high interest among medical professionals and the general public. An estimated 1.6 to 3.8 million cases of mild traumatic brain injury occur in sports and recreation each year in the United States, ${ }^{1}$ including nearly 63,000 concussions annually in high school sports. ${ }^{2}$ Although there are many grading scales and return-to-play guidelines, 3,4 clinicians and researchers have sought alternative methods to assess both the initial severity and ensuing recovery of the athletes under their care.

An important clinical component of patient follow-up involves the assessment of balance performance. The Balance Error Scoring System (BESS) has been shown to be a reliable and valid method in the assessment of balance performance. ${ }^{5}$ The BESS provides an adequate clinical representation of the balance deficits following concussion. ${ }^{6,7}$ As with any other type of injury, it would seem intuitive that concussion rehabilitation may return concussed athletes to play more safely; however, research in this area is lacking. Before such therapy can be adopted, there must be an investigation of the effects of concussion rehabilitation interventions in order to

Jason Mihalik is with the Department of Exercise and Sport Science, Mary C. Whitton and Luv Kohli are with the Department of Computer Science, all at the University of North Carolina, Chapel Hill. E-mail:jmihalik@email.unc.edu. 
better assess the long-term cognitive and physical outcomes in athletes with sportsrelated concussion. Because of the relatively quick recovery of many concussed athletes, one could argue that most athletes with concussion are not candidates for rehabilitation. It would seem reasonable, however, to intervene with rehabilitation in cases where the athlete experiences more prolonged symptoms, such as those with post-concussion syndrome. The literature is absent of any research findings relative to concussion rehabilitation in athletes, while only a few studies have investigated this in non-athletic populations and from the perspective of cognitive rehabilitation only. ${ }^{8,9}$ From the perspective of rehabilitation progression, it has long been understood that athletes should be progressed from simple, static, and slow activities to more challenging, dynamic, and functional tasks. ${ }^{10} \mathrm{~A}$ more recent study suggests that moderate exertion (physical and cognitive) within the initial few days following concussion may benefit the athlete's outcome. ${ }^{11}$ Thus, while more research is needed in the area of concussion rehabilitation, there is evidence to suggest that rehabilitation could benefit athletes following concussion.

Virtual environments (VE) technology may provide a rich opportunity to present both visual and cognitive stimuli to concussed athletes while, at the same time, protecting them from further physical harm. Immersive VEs may provide researchers a mechanism to better assess both balance and neurocognitive function simultaneously. A number of studies have investigated virtual reality in the guise of balance assessment and posturography; ${ }^{12,13}$ however, to our knowledge, only one study has investigated the use of virtual reality to assess balance performance in concussed athletes. ${ }^{14}$ Using a projection-based VE system, researchers demonstrated that concussed individuals, even 30 days after injury, exhibited impaired postural stability when presented with a moving visual field designed to induce self-motion. The work presented in this paper addresses balance in users wearing a head-mounted display (HMD). To date, no research has investigated the effects of the mass of the HMD on balance performance. Before we can use virtual environments to explore realistic and sport-specific stimuli to assess and rehabilitate athletes without placing them at risk of further injury, we need to know that the equipment itself does not decrease balance performance in healthy individuals. Therefore, the purpose of this study was to investigate the effects the mass of a HMD has on eyes-closed balance performance in healthy collegiate students.

\section{Methods}

This study used a counterbalanced design to assess the balance performance of healthy college students in two different conditions: while wearing a HMD (with integrated head-tracker sensor) and while wearing only the head-tracker sensor. Volunteer participants included 12 males and 8 females (height $=1.75 \pm 0.10 \mathrm{~m}$, mass $=76.42 \pm 16.5 \mathrm{~kg}$, age $=23.45 \pm 1.10 \mathrm{yrs}$ ). Participants had no history of head injury or vestibular disorders and were free of injury to the lower extremities for at least 3 months prior to data collection. Heavy drinkers, participants suffering from epilepsy, and those with symptoms of a cold were excluded from the study as these participants are at increased risk of adverse effects from VE immersion. All participants read and signed an informed consent form which had been approved by a university behavioral institutional review board. 


\section{Instrumentation}

Balance Error Scoring System. The BESS is a sideline measure of balance often used during preseason baseline testing and following a suspected sports-related concussion. The BESS consists of three stances performed on a firm surface: twolegged (A), one-legged (B), and tandem (C). These stances are repeated on a foam surface (D through F) and are depicted in Figure 1. All of the stances are performed with the eyes closed and last twenty seconds each. The foam surface consisted of medium-density foam measuring $40.0 \times 48.5 \times 6.5 \mathrm{~cm}$ (Airex Balance Pad, Alcan Airex AG, Switzerland). The BESS is scored by summing the observed number of errors (hands lifted off iliac crest; opening eyes; step, stumble, or fall; moving hip into $>30^{\circ}$ abduction; lifting forefoot or heel; remaining out of test position $>5 \mathrm{sec}$ ) during each 20 -second trial. High interrater reliability (coefficients ranging from 0.78 to 0.96$)^{5}$ and intrarater reliability (coefficients ranging from 0.87 to 0.98$)^{15}$ for the administration and evaluation of the BESS have been previously reported. Since the purpose of this study was to evaluate the effect of the mass of the HMD

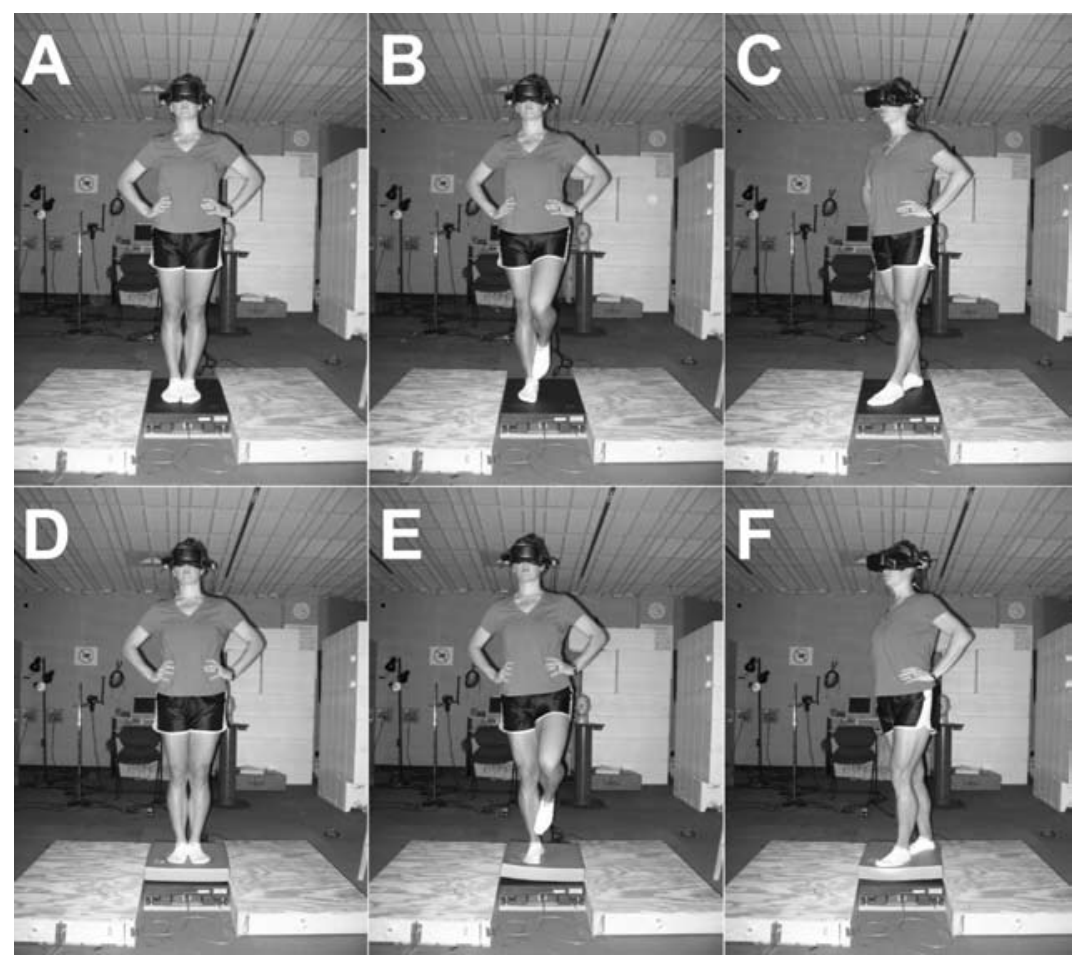

Figure 1 - The BESS consists of 6 stance positions including two-legged firm (stable) surface (A), one-legged firm surface (B), and tandem task on stable surface (C). These three stances are repeated on the foam surface (D-F). All trials are performed with the eyes closed. The figure depicts a participant performing the BESS while wearing the HMD unit. A spotter was present for all testing, but is not depicted in these photographs. 
on balance performance, an eyes-closed paradigm such as the BESS is a twofold appropriate tool: It eliminates the confounding variable of visual stimulus, and it allows us to relate our findings to a commonly used concussion assessment tool (BESS).

Force Platform. The BESS was performed on a Bertec 4060-08 piezoelectric crystal force platform (Bertec Corp., Columbus, $\mathrm{OH}$ ). The Bertec force platform is interfaced with a personal desktop computer via a 16-bit analog to digital (A/D) converter board. Raw force platform data was collected using The MotionMonitor for Research Software Version 6.74 (Innovative Sports Training, Inc., Chicago, IL). Force platform data were collected at a sampling frequency of $1000 \mathrm{~Hz}$.

Head-Mounted Display. Video was presented to the participants with a Virtual Research V8 HMD at a resolution of $640 \times 480$ pixels in each eye and a 60 -degree diagonal field of view. The HMD's video refresh rate was $60 \mathrm{~Hz}$. A 3rdTech HiBall $^{\mathrm{TM}} 3000$ head tracker was also used to provide position and orientation data of the head at a frequency of $600 \mathrm{~Hz}$. The dimensions of the HMD were 44.45 $\times 20.32 \times 15.24 \mathrm{~cm}$ and the combined mass of the HMD and HiBall tracker was $1.13 \mathrm{~kg}$; the mass of the tracker alone is $0.17 \mathrm{~kg}(6 \mathrm{oz}$.). The equipment is depicted in Figure 2. The visual content presented in the HMD was set to mimic reality so that when subjects opened their eyes (which is a documented BESS error), they received relatively accurate information about where they were in space. Data from the HiBall tracker were used by the system to generate the view appropriate to the user's current point-of-view.

\section{Interventions}

The testing procedures were explained to participants prior to arrival at the virtual reality laboratory. There were two testing conditions: BESS with only the tracker and BESS while wearing the HMD (including tracker). The conditions were counterbalanced. To mimic the clinical application of the BESS, we tested each participant under the six different balance tasks of the BESS. The three firm-surfaced tasks were performed directly on the force platform; for the three foam-surfaced tasks,

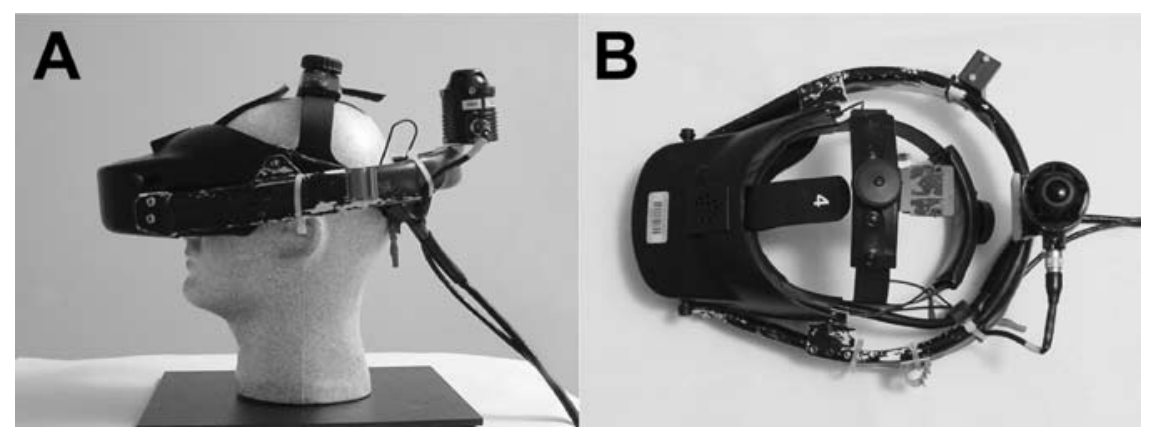

Figure 2 - Lateral (A) and superior (B) views of the HMD as it rests on a generic headform. 
participants stood on medium-density foam that was placed directly on the force platform. Each trial lasted 20 seconds and errors were counted. The participants completed one series of the BESS under each counterbalanced condition, with a 5 minute rest period between the two. During the condition when the participants wore the HMD, they were asked to self-report the number of times they opened their eyes at the conclusion of each of the six BESS balance conditions. The experimenters positioned themselves near the subject during the task in case the subject stumbled or fell while on the platform.

\section{Outcome Measures}

The BESS results in a single total error score (higher scores reflect poor performance) for all six balance tasks combined. We also wanted to investigate the effects of stance (two-legged, one-legged, and tandem) as well as surface (firm and foam); therefore, error totals by stance and surface were also computed. Force platform data was filtered within The MotionMonitor for Research software with a fourth-order zero-phase lag low pass Butterworth digital filter with a cut-off frequency of $20 \mathrm{~Hz} .{ }^{16}$ We exported the center of pressure (COP) values from The MotionMonitor for Research software to Matlab 7 (The Mathworks, Inc.; Natick, MA) in order to calculate sway area and travel distance for each 20-second trial. The sway area, which is often used to determine the extent of postural stability, was calculated by the minor and major axes of an ellipse that statistically encompassed an area containing $95 \%$ of the center of pressure data points. Previous researchers have used the elliptical method for determining postural sway area, ${ }^{17-19}$ and the procedure for calculating a $95 \%$ confidence ellipse has been well described. ${ }^{20}$ The travel distance is defined as the length of the path of the COP movement over the testing time period. This has been a very common measure in the assessment of balance performance.

\section{Statistical Analyses}

We performed an a priori sample size estimate based on previously published literature comparing differences in balance performance between concussed athletes and healthy controls. Based on an effect size of 1.61 for foam-surfaced conditions, ${ }^{21}$ we would need 8 subjects in our study to identify any clinically significant changes in balance performance with a power of 0.80 . While we acknowledge studying healthy participants in this study, we chose to double this sample size to study at least 16 participants to be conservative. To test our primary research question of whether the use of a HMD adversely affects balance performance in healthy individuals, we performed a repeated measures analysis of variance with 3 within-subjects factors: HMD condition (on or off), BESS surface (firm or foam), and BESS stance (two-legged, one-legged, or tandem) on each of the following outcome measures: BESS error, sway area, and travel distance. All statistical analyses were performed in SPSS 13 (SPSS Inc.; Chicago, IL) and the level of significance was set at $P<$ .05 a priori. When there were significant main effects, post hoc analyses using Tukey's $H S D$ were performed on the data to correct for multiple comparisons. We also calculated effect sizes for each of the testing conditions in order to assess the clinical significance of our findings in the event of a $P>0.05$ statistical result. 


\section{Results}

We performed the BESS on 20 healthy individuals. Force platform data from 4 subjects were rejected prior to analysis due to recording failures. Therefore, we report the BESS findings based on 20 participants and include analyses of our force platform measures based on 16 participants. This did not affect our overall findings; results from two participants in each test order were rejected. This still allowed for an equally counterbalanced design for the remaining 16 participants. Descriptive data for the separate conditions, surfaces, and stances are presented in Tables 1 and 2. Our computations revealed very low effect sizes for BESS error scores. These are presented in Table 3.

We did not observe a 3-way interaction for the effects of the HMD on BESS performance $\left(F_{1.54,29.34}=0.65, P=0.492\right)$. We did reveal a significant 2 -way interaction for surface and stance $\left(F_{2,38}=38.72, P<0.001\right)$. We did not find 2-way interactions for HMD and stance $\left(F_{1.58,24.09}=1.24, P=0.294\right)$ and for HMD and surface $\left(F_{1,19}=0.52, P=0.479\right)$. Simple main effects testing revealed no significant differences in BESS error scores regardless of whether the participant wore

Table 1 Mean (SD) Balance Error Scores, Elliptical Sway Area, and COP Distance Traveled While Performing the BESS Without the HMD.

\begin{tabular}{cccc}
\hline Balance Condition & BESS Error Scores & Sway Area, $\mathbf{c m}^{\mathbf{2}}$ & $\begin{array}{c}\text { COP Distance, } \\
\mathbf{c m}\end{array}$ \\
\hline A & 0 & $6.96(4.40)$ & $43.17(10.72)$ \\
B & $1.75(1.29)$ & $61.87(68.37)$ & $218.00(109.74)$ \\
C & $0.45(0.51)$ & $19.85(20.51)$ & $121.29(46.17)$ \\
D & $0.05(0.22)$ & $30.37(14.20)$ & $124.88(42.41)$ \\
E & $5.20(1.67)$ & $167.19(106.65)$ & $347.87(126.26)$ \\
F & $2.75(1.62)$ & $108.11(90.57)$ & $282.32(159.56)$ \\
Total & $10.20(3.38)$ & N/A & N/A \\
\hline
\end{tabular}

Note. Balance conditions A-F refer to those identified in Figure 1.

Table 2 Mean (SD) Balance Error Scores, Elliptical Sway Area, and COP Distance Traveled While Performing the BESS With the HMD

\begin{tabular}{cccc}
\hline Balance Condition & BESS Error Scores & Sway Area, $\mathbf{c m}^{2}$ & COP Distance, $\mathbf{c m}$ \\
\hline A & 0 & $7.74(4.16)$ & $47.84(17.37)$ \\
B & $2.35(1.76)$ & $37.40(23.82)$ & $187.63(56.97)$ \\
C & $0.50(0.83)$ & $18.24(16.64)$ & $112.69(26.25)$ \\
D & $0.05(0.22)$ & $29.09(12.45)$ & $113.40(34.51)$ \\
E & $5.35(2.46)$ & $116.53(41.84)$ & $302.76(73.91)$ \\
F & $2.85(1.76)$ & $94.57(80.67)$ & $246.15(121.33)$ \\
Total & $11.10(4.70)$ & N/A & N/A \\
\hline
\end{tabular}

Note. Balance conditions A-F refer to those identified in Figure 1. 
Table 3 Calculated Effect Sizes (ES) for BESS Error Scores, Elliptical Sway Area, and COP Distance Traveled for Each Balance Condition

\begin{tabular}{cccc}
\hline Balance Condition & BESS ES & Sway Area ES & COP Distance ES \\
\hline A & 0 & 0.18 & 0.33 \\
B & 0.46 & 0.53 & 0.36 \\
C & 0.10 & 0.09 & 0.24 \\
D & 0 & 0.1 & 0.3 \\
E & 0.09 & 0.68 & 0.45 \\
F & 0.06 & 0.16 & 0.26 \\
Total & 0.22 & N/A & N/A \\
\hline
\end{tabular}

Note. Balance conditions A-F refer to those identified in Figure 1.

the HMD or not $\left(F_{1,19}=1.71, P=0.206\right)$. Significant main effects were noted for surface $\left(F_{1,19}=119.41, P<0.001\right)$ with mean error scores higher in balance tasks performed on foam (2.71) compared to firm surfaces (0.84). Further, significant main effects for stance were also observed $\left(F_{1.65,31.39}=73.11, P<0.001\right)$. Post hoc analyses revealed higher error scores recorded during one-legged tasks (3.66) compared to tandem (1.64) and two-legged (0.03) stances.

The analyses run on the elliptical sway area and travel distance outcome measures generated from the force platform COP values yielded similar findings to the BESS. No significant 3-way interaction between HMD condition, surface, and stance was observed in our measure of elliptical sway area $\left(F_{2,18}=0.91\right.$, $P=0.422$ ). We observed a significant 2 -way interaction for surface and stance $\left(F_{2,18}=5.99, P=0.01\right)$. However, we did not find 2-way interactions for HMD and stance $\left(F_{2,18}=1.66, P=0.218\right)$ and for HMD and surface $\left(F_{1,9}=0.48, P=0.505\right)$. We observed significant main effects for surface $\left(F_{1,19}=47.13, P<0.001\right)$, with participants' sway area increasing in balance tasks performed on foam $\left(87.37 \mathrm{~cm}^{2}\right)$ compared to firm surfaces $\left(21.44 \mathrm{~cm}^{2}\right)$. Further, significant main effects for stance were also observed $\left(F_{2,18}=11.26, P=0.001\right)$. Post hoc analyses noted that sway area during one-legged $\left(90.32 \mathrm{~cm}^{2}\right)$ and tandem tasks $\left(56.86 \mathrm{~cm}^{2}\right)$ were significantly higher than the sway area observed during two-legged stance $\left(16.04 \mathrm{~cm}^{2}\right)$; no statistically significant differences in sway area were observed between onelegged and tandem stances. Simple main effects testing revealed no significant differences in sway area regardless of whether the participant wore the HMD or $\operatorname{not}\left(F_{1,9}=1.16, P=0.310\right)$.

Findings related to COP travel distance presented similar trends. Again, no significant 3-way interaction was observed in our measure of travel distance between HMD condition, surface, and stance $\left(F_{1.22,10.99}=0.132, P=0.772\right)$. Similarly, no significant 2-way interactions were observed for HMD and stance $\left(F_{1.40,12.63}=0.95, P=0.380\right)$ and for HMD and surface $\left(F_{1,9}=0.86, P=0.378\right)$ for COP travel distance; however, we also found a significant 2-way interaction for surface and stance $\left(F_{128,11.49}=4.75, P=0.044\right)$. Significant main effects for surface $\left(F_{1,9}=55.00, P<0.001\right)$ were observed; the distance traveled by the COP was 
significantly longer in foam-surfaced conditions $(232.91 \mathrm{~cm})$ compared to tasks performed on a firm surface $(113.95 \mathrm{~cm})$. A significant main effect was noted for stance $\left(F_{2,18}=30.52, P<0.001\right)$, with participants' COP distance traveled significantly higher in one-legged $(255.76 \mathrm{~cm})$ and tandem $(188.78 \mathrm{~cm})$ stances as compared to two-legged stance $(75.76 \mathrm{~cm})$; one-legged stance demonstrated significantly higher COP distance traveled than tandem stance. Simple main effects testing revealed no observable differences in COP travel distance based on HMD condition $\left(F_{1,9}=1.17, P=0.308\right)$.

\section{Comments}

To our knowledge, this study was the first to investigate the effects of the mass of a HMD on balance performance in an attempt to assess the potential for using virtual reality in future research involving concussed athletes. The results of our ANOVA do not let us reject the null hypothesis that the mass of the HMD would have no impact on balance performance. However, an analysis of the effect sizes of these comparisons demonstrate very low clinical differences between the two conditions; these differences are far less than what has been previously observed in the literature that has compared concussed athletes to healthy control participants. Taken together, these data suggest that the mass of the HMD itself has little influence on the use of virtual environments for the evaluation of balance in a healthy control population.

\section{Balance Error Scoring System}

The BESS has been used extensively in concussion research and is currently part of many concussion assessment protocols. The BESS protocol is a widely used and applicable sideline field test used to assess postural control following a suspected mild traumatic brain injury. As the applicability of virtual reality research in the area of sports-related concussion continues to be explored, assessing the potential confounding influence of a HMD's mass on a participant's ability to maintain postural control is a very important research question. Our study results support the findings of previous research ${ }^{5,22,23}$ on base-of-support: tasks performed on the foam surface elicited inferior balance performance than those performed on a stable surface.

Stance can also be a confounder. Balance performance in our study was diminished during one-legged stance. This is in agreement with previous literature..$^{524-26}$ This is believed to occur due to the body's reorganization of the center of mass over a smaller base of support. This increased difficulty in maintaining single-leg steadiness may better identify postural control differences that exist when comparing populations such as those with mild head injuries and healthy individuals and is an important reason why this stance was included as the BESS was developed. Activities of daily living such as reaching for high objects and the swing phase of gait, are often better replicated by single-leg tasks. In orthopedic settings, it is often beneficial to perform single-leg assessments since this allows for bilateral comparisons within subjects. ${ }^{5}$ 


\section{Force Platform Measures}

Force platforms have been used to evaluate balance performance in the past. ${ }^{27,28} \mathrm{~A}$ number of tasks have been studied such as two-legged, tandem, step, and one-legged stances. ${ }^{21,28}$ All have been shown to be increasingly difficult with foam surfaces and with the subjects' eyes closed; however, fixed and stable surfaces are the most commonly used in the measurement of postural stability. Another finding in our study was the similarity of statistical findings between elliptical sway area, COP travel distance (force platform measures), and the BESS error scores. Specifically, our force platform measures confirmed our BESS findings that larger deficits in balance were observed while performing foam-surfaced tasks and that the one-legged task remained more difficult on foam than tandem or two-legged tasks. These results agree with previously published reports in the literature..$^{28,29}$

\section{Head-Mounted Display}

Perhaps the most interesting and novel component to this study was the use of a HMD while performing a clinical balance performance assessment. As stated earlier, there were no significant effects of the HMD mass. The observed two-way interactions between surface and stance were negated when the use of a HMD was entered into the model. These results affirm that the mass of the HMD has not been proven to adversely affect balance performance in healthy individuals. This is a very promising finding as future studies with concussed athletes will continue to develop the possibility of incorporating virtual environments into the concussion research paradigm. This is especially true in the context of investigating balance performance queries.

Although our observed statistical power was moderate at best, we also calculated effect sizes that were extremely low (Table 3 ). This suggests that some of our findings, although potentially nonsignificant due to limited sample size, were not clinically significant. Differences in BESS errors between concussed and healthy athletes typically yield high effect sizes, and both clinically and statistically significant differences. Wearing a HMD while performing the BESS resulted in a mean 0.9 error increase and yielded an effect size of 0.22 (Table 3). Based on a paired-sample model estimate, we would need in excess of 160 participants in order to have our observed mean total error increase yield a statistically significant difference. Based on previously published literature and our extensive experience with the BESS, a 0.9 mean error increase would yield clinically insignificant findings. Riemann et $\mathrm{al},{ }^{21}$ for example, investigated the difference in BESS balance performance between athletes suffering from mild head injury and healthy controls; they observed a mean increase of 9 recorded errors in the injured participants compared to the performance of the healthy comparison sample. Furthermore, the mean BESS error difference between the two test conditions in our study was smaller than differences observed in the literature comparing concussed athletes to healthy controls as far as 3 days following injury. ${ }^{30}$

\section{Limitations}

Although the HMD offers researchers a rich opportunity to provide customizable and modifiable visual stimuli to study participants, there are some limitations in 
the implementation used for this study. In order to decrease the demands on the tracking and rendering systems, a virtual model of the body is not present. For example, when a person wearing a HMD looks down to the floor, they will see the virtual model of the force platform but are unable to view the position of their feet. As a result, our subjects described slight apprehension of performing the balance tasks while wearing the HMD.

We recognize that there are some limitations to our study design that are worth discussing. Since we did not control for the level of physical activity of our participants, this may limit the extension of our findings to all young athletes. Another limitation to our study was the lack of adolescent participants. Understanding the manifestations of concussion in this population has been an area of interest presented in the recent literature. Future studies should identify whether the HMD poses an external influence on balance performance in adolescents as they may lack the physical stature to overcome the mass of the HMD. Future studies should also incorporate a number of balance conditions with the eyes open to assess the influence of visual stimuli in virtual environments on balance performance.

The use of virtual environments in better understanding the effects of sportsrelated concussion is an important advance in this area of research. The large number of publications in the area of sports-related concussion has done well to elucidate the deficits expected in athletes following a mild head injury. It is also generally accepted that athletes be withheld from activity until symptoms and objective measures of concussion, including balance performance, return to preinjury levels. Unfortunately, many certified athletic trainers working with high school and collegiate athletes must often find a healthy medium by which cognitive exertion (eg, attending classes) will not prolong recovery. Research in this area is anecdotal at best; we believe virtual environments may provide an excellent venue in which to expose an injured athlete to the physical stimuli of their sport without placing them in inherent danger of physical contact that may lead to potentially serious secondary injuries such as second impact syndrome. In theory, a virtual environment can be modified to reflect the current reactive state of an injured athlete as it is measured by conventional neurocognitive testing. We believe that virtual environments may be a mechanism by which we can improve an injured athlete's functional and sport-specific reaction time prior to returning them to play. In cases where symptoms and cognitive decline persist, we feel that virtual environments may provide an arena to rehabilitate an athlete not only by exposing him or her to physical demands associated with their sport, but also by targeting cognitive functions needed to succeed in the classroom.

\section{References}

1. Langlois JA, Rutland-Brown W, Wald MM. The epidemiology and impact of traumatic brain injury: a brief overview. J Head Trauma Rehabil. 2006;21:375-378.

2. Powell JW, Barber-Foss KD. Traumatic brain injury in high school athletes. JAMA. 1999;282:958-963.

3. Johnston KM, McCrory P, Mohtadi NG, Meeuwisse W. Evidence-Based review of sport-related concussion: clinical science. Clin J Sport Med. 2001;11:150-159.

4. Leclerc S, Lassonde M, Delaney JS, Lacroix VJ, Johnston KM. Recommendations for grading of concussion in athletes. Sports Med. 2001;31:629-636. 
5. Riemann BL, Guskiewicz KM, Shields EW. Relationship between clinical and forceplate measures of postural stability. J Sport Rehabil. 1999;8:71-82.

6. Guskiewicz KM, Perrin DH. Research and clinical applications of assessing balance. J Sport Rehabil. 1996;5:45-63.

7. Guskiewicz KM, Riemann BL, Perrin DH, Nashner LM. Alternative approaches to the assessment of mild head injury in athletes. Med Sci Sports Exerc. 1997;29:S213221.

8. Salazar AM, Warden DL, Schwab K, et al. Cognitive rehabilitation for traumatic brain injury: a randomized trial. Defense and Veterans Head Injury Program (DVHIP) study group. JAMA. 2000;283:3075-3081.

9. Warden DL, Salazar AM, Martin EM, Schwab KA, Coyle M, Walter J. A home program of rehabilitation for moderately severe traumatic brain injury patients. The DVHIP study group. J Head Trauma Rehabil. 2000;15:1092-1102.

10. Guskiewicz KM. Regaining balance and postural equilibrium. In: Prentice WE, ed. Rehabilitation Techniques in Sports medicine. 3rd ed. Boston, MA: WCB McGrawHill; 1999.

11. Majerske CW, Mihalik JP, Ren D, et al. Concussion in sports: the effect of post-concussive activity levels on symptoms and neurocognitive performance. J Athl Train. 2008;In press.

12. Bisson E, Contant B, Sveistrup H, Lajoie Y. Functional balance and dual-task reaction times in older adults are improved by virtual reality and biofeedback training. Cyberpsychol Behav. 2007;10:16-23.

13. Thornton M, Marshall S, McComas J, Finestone H, McCormick A, Sveistrup H. Benefits of activity and virtual reality based balance exercise programmes for adults with traumatic brain injury: perceptions of participants and their caregivers. Brain Inj. 2005; 19:989-1000.

14. Slobounov S, Slobounov E, Newell K. Application of virtual reality graphics in assessment of concussion. Cyberpsychol Behav. 2006;9:188-191.

15. Valovich McLeod TC, Perrin DH, Guskiewicz KM, Shultz SJ, Diamond R, Gansneder BM. Serial administration of clinical concussion assessments and learning effects in healthy young athletes. Clin J Sport Med. 2004;14:287-295.

16. Horak FB, Hlavacka F. Vestibular stimulation affects medium latency postural muscle responses. Exp Brain Res. 2002;144:95-102.

17. Kuo AD, Speers RA, Peterka RJ, Horak FB. Effect of altered sensory conditions on multivariate descriptors of human postural sway. Exp Brain Res. 1998;122:185-195.

18. Nault ML, Allard P, Hinse S, et al. Relations between standing stability and body posture parameters in adolescent idiopathic scoliosis. Spine. 2002;27:1911-1917.

19. Prieto TE, Myklebust JB, Hoffmann RG, Lovett EG, Myklebust BM. Measures of postural steadiness: differences between healthy young and elderly adults. IEEE Trans Biomed Eng. 1996;43:956-966.

20. Sokal RR, Rohlf FJ. Biometry: The Principles and Practice of Statistics in Biological Research. 3rd ed. New York: W.H. Freeman and Company; 1995.

21. Riemann BL, Guskiewicz KM. Effects of mild head injury on postural stability as measured through clinical balance testing. J Athl Train. 2000;35:19-25.

22. Day BL, Steiger MJ, Thompson PD, Marsden CD. Effect of vision and stance width on human body motion when standing: implications for afferent control of lateral sway. J Physiol. 1993;469:479-499.

23. Kirby RL, Price NA, MacLeod DA. The influence of foot position on standing balance. J Biomech. 1987;20:423-427.

24. Hasan SS, Lichtenstein MJ, Shiavi RG. Effect of loss of balance on biomechanics platform measures of sway: influence of stance and a method for adjustment. $J$ Biomech. 1990;23:783-789. 
25. Wilkins JC, Valovich McLeod TC, Perrin DH, Gansneder BM. Performance on the balance error scoring system decreases after fatigue. J Athl Train. 2004;39:156-161.

26. Susco TM, Valovich McLeod TC, Gansneder BM, Shultz SJ. Balance recovers within 20 minutes after exertion as measured by the balance error scoring system. J Athl Train. 2004;39:241-246.

27. Murray MP, Seireg AA, Sepic SB. Normal postural stability and steadiness: quantitative assessment. J Bone Joint Surg Am. 1975;57:510-516.

28. Goldie PA, Bach TM, Evans OM. Force platform measures for evaluating postural control: reliability and validity. Arch Phys Med Rehabil. 1989;70:510-517.

29. Goldie PA, Evans OM, Bach TM. Steadiness in one-legged stance: development of a reliable force-platform testing procedure. Arch Phys Med Rehabil. 1992;73:348-354.

30. McCrea M, Guskiewicz KM, Marshall SW, et al. Acute effects and recovery time following concussion in collegiate football players: the NCAA concussion study. JAMA. 2003;290:2556-2563. 